TAIWANESE JOURNAL OF MATHEMATICS

Vol. 11, No. 5, pp. 1431-1441, December 2007

This paper is available online at http://www.math.nthu.edu.tw/tjm/

\title{
A CHARACTERIZATION OF TWO-SIDED CENTRALIZERS ON PRIME RINGS
}

\author{
Joso Vukman and Maja Fošner
}

\begin{abstract}
In this paper we prove the following result: Let $R$ be a prime ring of characteristic different from two and let $T: R \rightarrow R$ be an additive mapping satisfying the relation $T\left(x^{3}\right)=x T(x) x$ for all $x \in R$. In this case $T$ is a two-sided centralizer.
\end{abstract}

\section{INTRODUCTION}

Throughout the paper $R$ will represent an associative ring. A ring $R$ is 2 -torsion free if $2 x=0, x \in R$, implies $x=0$. Recall that $R$ is a semiprime ring if $a R a=0$ (where $a \in R$ ) implies $a=0$, and it is called a prime ring if $a R b=0$ (where $a, b \in R$ ) implies $a=0$ or $b=0$. An additive mapping $D: R \rightarrow R$ is called a derivation if $D(x y)=D(x) y+x D(y)$ for all $x, y \in R$, and is called a Jordan derivation if $D\left(x^{2}\right)=D(x) x+x D(x)$ for all $x \in R$. Obviously, any derivation is a Jordan derivation. The converse is in general not true. A classical result of Herstein [10] asserts that any Jordan derivation on a prime ring with characteristic different from two is a derivation. Cusack [9] generalized Herstein's result to 2torsion free semiprime rings (see also [8] for an alternative proof). An additive mapping $T: R \rightarrow R$ is called a left centralizer if $T(x y)=T(x) y$ for all $x, y \in R$. The concept appears naturally in $C^{*}$-algebras. In ring theory it is more common to work with module homomorphisms. Ring theorists would write that $T: R_{R} \rightarrow R_{R}$ is a homomorphism of the right $R$-module $R$ into itself. For a semiprime ring $R$ all such homomorphisms are of the form $T(x)=q x$ for all $x \in R$, where $q$ is an element of Martindale right ring of quotients $Q_{r}$. For the explanation of $Q_{r}, Q_{s}$ (the symmetric Martindale ring of quotients), and $Q_{m r}$ (the maximal right ring of

Accepted January 23, 2006.

Communicated by Wen-Fong Ke.

2000 Mathematics Subject Classification: 16N60, 39B05.

Key words and phrases: Prime ring, Semiprime ring, Functional identity, Left (right) centralizer, Two-sided centralizer. 
quotients) see [5, Chapter 2]. In case $R$ has the identity element $T: R \rightarrow R$ is a left centralizer if and only if $T$ is of the form $T(x)=a x$ for all $x \in R$ and some fixed element $a \in R$. A left Jordan centralizer $T: R \rightarrow R$ is an additive mapping such that $T\left(x^{2}\right)=T(x) x$ for all $x \in R$. The definition of a right centralizer and a right Jordan centralizer should be self-explanatory. An additive mapping $T: R \rightarrow R$ is called a two-sided centralizer if $T$ is a left and a right centralizer. In case $T: R \rightarrow R$ is a two-sided centralizer, where $R$ is a semiprime ring with extended centroid $C$, then there exists $\lambda \in C$ such that $T(x)=\lambda x$ for all $x \in R$ (see [1, Theorem 2.3.2]). Following ideas from [8] Zalar [17] proved that any left (right) Jordan centralizer on a 2-torsion free semiprime ring is a left (right) centralizer. Vukman [14] proved that an additive mapping $T: R \rightarrow R$, where $R$ is a 2-torsion free semiprime ring, satisfying $2 T\left(x^{2}\right)=T(x) x+x T(x)$ for all $x \in R$, is a two-sided centralizer. Molnár [11] proved that an additive mapping $T: A \rightarrow A$, where $A$ is a semisimple $H^{*}$-algebra, satisfying the relation $T\left(x^{3}\right)=T(x) x^{2}$ (resp. $T\left(x^{3}\right)=x^{2} T(x)$ ) for all $x \in A$, is a left (resp. right) centralizer. Our aim in this paper is to consider an additive mapping $T$ from a prime ring $R$ of characteristic different from two into itself such that the identity $T\left(x^{3}\right)=x T(x) x$ holds true for all $x \in R$. Our result is obtained as an application of the theory of functional identities.

The theory of functional identities considers set-theoretic mappings on rings satisfying some identical relations. When treating such relations one usually concludes that the form of the mappings involved can be described, unless the ring is very special. We refer the reader to [7] for introductory account on functional identities, where Brešar presents this new theory, the theory of (generalized) functional identities, and its applications, to a wider audience.

Let $R$ be a ring and let $X$ be a subset of $R$. As usual the commutator $x y-y x$, $x, y \in R$, will be denoted by $[x, y]$. By $C(X)$ we denote the set $\{r \in R \mid[r, X]=$ $0\}$. Let $m \in \mathbb{N}$ and let $E: X^{m-1} \rightarrow R, p: X^{m-2} \rightarrow R$ be arbitrary mappings. In the case when $m=1$ this should be understood as that $E$ is an element in $R$ and $p=0$. Let $1 \leq i<j \leq m$ and define $E^{i}, p^{i j}, p^{j i}: X^{m} \rightarrow R$ by

$$
\begin{aligned}
E^{i}\left(\bar{x}_{m}\right) & =E\left(x_{1}, \ldots, x_{i-1}, x_{i+1}, \ldots, x_{m}\right), \\
p^{i j}\left(\bar{x}_{m}\right)=p^{j i}\left(\bar{x}_{m}\right) & =\left(x_{1}, \ldots, x_{i-1}, x_{i+1}, \ldots, x_{j-1}, x_{j+1}, \ldots, x_{m}\right),
\end{aligned}
$$

where $\bar{x}_{m}=\left(x_{1}, \ldots, x_{m}\right) \in X^{m}$.

Let $I, J \subseteq\{1, \ldots, m\}$, and for each $i \in I, j \in J$ let $E_{i}, F_{j}: X^{m-1} \rightarrow R$ be arbitrary mappings. Consider functional identities

$$
\begin{array}{cc}
\sum_{i \in I} E_{i}^{i}\left(\bar{x}_{m}\right) x_{i}+\sum_{j \in J} x_{j} F_{j}^{j}\left(\bar{x}_{m}\right)=0 & \left(\bar{x}_{m} \in X^{m}\right), \\
\sum_{i \in I} E_{i}^{i}\left(\bar{x}_{m}\right) x_{i}+\sum_{j \in J} x_{j} F_{j}^{j}\left(\bar{x}_{m}\right) \in C(X) & \left(\bar{x}_{m} \in X^{m}\right) .
\end{array}
$$


A natural possibility when (1) and (3) are fulfilled is when there exist mappings $p_{i j}: X^{m-2} \rightarrow R, i \in I, j \in J, i \neq j$, and $\lambda_{k}: X^{m-1} \rightarrow C(X), k \in I \cup J$, such that

$$
\begin{aligned}
& E_{i}^{i}\left(\bar{x}_{m}\right)=\sum_{j \in J, j \neq i} x_{j} p_{i j}^{i j}\left(\bar{x}_{m}\right)+\lambda_{i}^{i}\left(\bar{x}_{m}\right), \\
& F_{j}^{j}\left(\bar{x}_{m}\right)=-\sum_{i \in I, j \neq i} p_{i j}^{i j}\left(\bar{x}_{m}\right) x_{i}-\lambda_{j}^{j}\left(\bar{x}_{m}\right), \\
& \lambda_{k}=0 \quad \text { if } \quad k \notin I \cap J
\end{aligned}
$$

for all $\bar{x}_{m} \in X^{m}, i \in I, j \in J$. We shall say that every solution of the form (3) is a standard solution of (1) and (2).

The case when one of the sets $I$ or $J$ is empty is not excluded. The sum over the empty set of indexes should be simply read as zero. So, when $J=0$ (resp. $I=0)(1)$ and (2) reduce to

$$
\begin{gathered}
\sum_{i \in I} E_{i}^{i}\left(\bar{x}_{m}\right) x_{i}=0 \quad\left(\text { resp. } \sum_{j \in J} x_{j} F_{j}^{j}\left(\bar{x}_{m}\right)=0\right) \quad\left(\bar{x}_{m} \in X^{m}\right), \\
\sum_{i \in I} E_{i}^{i}\left(\bar{x}_{m}\right) x_{i} \in C(X) \quad\left(\text { resp. } \sum_{j \in J} x_{j} F_{j}^{j}\left(\bar{x}_{m}\right) \in C(X)\right) \quad\left(\bar{x}_{m} \in X^{m}\right) .
\end{gathered}
$$

In that case the (only) standard solution is

$$
E_{i}=0, \quad i \in I \quad\left(\text { resp. } F_{j}=0, \quad j \in J\right) .
$$

A $d$-freeness of $X$ will play an important role in this paper. For a definition of $d$-freeness we refer the reader to [6]. Under some natural assumptions one can establish that various subsets (such as ideals, Lie ideals, the sets of symmetric or skew symmetric elements in a ring with involution) of certain types of rings are $d$-free. We refer the reader to [1] and [2] for results of this kind. Let us mention that a prime ring $R$ is a $d$-free subset of its maximal right ring of quotients, unless $R$ satisfies the standard polynomial identity of degree less than $2 d$ (see [2, Theorem 2.4]).

\section{RESULTS}

Motivated by results on centralizers and derivations, Vukman proved the following result.

Theorem 2.1. Let $R$ be a 2-torsion free semiprime ring and let $T: R \rightarrow R$ be an additive mapping satisfying the relation

$$
T(x y x)=x T(y) x
$$


for all $x, y \in R$. Then $T$ is a two-sided centralizer.

Puting $x=y$ in (7) we obtain

$$
T\left(x^{3}\right)=x T(x) x
$$

for all $x \in R$. Our aim in this paper is to prove the following result which is related to $(8)$.

Theorem 2.2. Let $R$ be a prime ring of characteristic different from two and let $T: R \rightarrow R$ be an additive mapping satisfying the relation

$$
T\left(x^{3}\right)=x T(x) x
$$

for all $x \in R$. Then $T$ is a two-sided centralizer.

Let us point out that in this paper we use methods which differs from those used in [15]. The proof of Theorem 2.1 is elementary in the sense that one needs no specific knowledge concerning semiprime rings in order to follow the proof. In this paper we use methods and results from the theory of functional identities.

Before proving Theorem 2.2 we need one more result. Let $R$ be a ring and let

$$
p\left(x_{1}, x_{2}, x_{3}\right)=\sum_{\pi \in S_{3}} x_{\pi(1)} x_{\pi(2)} x_{\pi(3)}
$$

be a fixed multilinear polynomial in noncommuting indeterminates $x_{1}, x_{2}$ and $x_{3}$. Further, let $L$ be a subset of $R$ closed under $p$, i.e. $p\left(\bar{x}_{3}\right) \in L$ for all $x_{1}, x_{2}, x_{3} \in L$, where $\bar{x}_{3}=\left(x_{1}, x_{2}, x_{3}\right)$. We shall consider a mapping $T: L \rightarrow R$ satisfying

$$
T\left(p\left(\bar{x}_{3}\right)\right)=\sum_{\pi \in S_{3}} x_{\pi(1)} T\left(x_{\pi(2)}\right) x_{\pi(3)}
$$

for all $x_{1}, x_{2}, x_{3} \in L$. Of course, every two-sided centralizer satisfies (9). Our goal is to show that under certain assumptions these are in fact the only mappings with this property. In the first step of the proof we derive a functional identity from (9). Let us mention that the idea of considering the expression $\left[p\left(\bar{x}_{3}\right), p\left(\bar{y}_{3}\right)\right]$ in its proof is taken from [4].

Theorem 2.3. Let $L$ be a 6-free Lie subring of $R$ closed under $p$. If $T$ : $L \rightarrow R$ is an additive mapping satisfying (9), then there exists $\lambda \in C(L)$ such that $T(x)=\lambda x$ for all $x \in L$.

Proof. Note that for any $a \in R$ and $\bar{x}_{3} \in L^{3}$ we have

$$
\left[p\left(\bar{x}_{3}\right), a\right]=p\left(\left[x_{1}, a\right], x_{2}, x_{3}\right)+p\left(x_{1},\left[x_{2}, a\right], x_{3}\right)+p\left(x_{1}, x_{2},\left[x_{3}, a\right]\right) .
$$


Thus

$T\left[p\left(\bar{x}_{3}\right), a\right]=T\left(p\left(\left[x_{1}, a\right], x_{2}, x_{3}\right)\right)+T\left(p\left(x_{1},\left[x_{2}, a\right], x_{3}\right)\right)+T\left(p\left(x_{1}, x_{2},\left[x_{3}, a\right]\right)\right)$.

Using (9) it follows that

$$
\begin{aligned}
& T\left[p\left(\bar{x}_{3}\right), a\right]=\sum_{\pi \in S_{3}}\left[x_{\pi(1)}, a\right] T\left(x_{\pi(2)}\right) x_{\pi(3)} \\
& \quad+\sum_{\pi \in S_{3}} x_{\pi(1)} T\left[x_{\pi(2)}, a\right] x_{\pi(3)}+\sum_{\pi \in S_{3}} x_{\pi(1)} T\left(x_{\pi(2)}\right)\left[x_{\pi(3)}, a\right] .
\end{aligned}
$$

In particular

$$
\begin{aligned}
T\left[p\left(\bar{x}_{3}\right), p\left(\bar{y}_{3}\right)\right]= & \sum_{\pi \in S_{3}}\left[x_{\pi(1)}, p\left(\bar{y}_{3}\right)\right] T\left(x_{\pi(2)}\right) x_{\pi(3)} \\
& +\sum_{\pi \in S_{3}} x_{\pi(1)} T\left[x_{\pi(2)}, p\left(\bar{y}_{3}\right)\right] x_{\pi(3)} \\
& +\sum_{\pi \in S_{3}} x_{\pi(1)} T\left(x_{\pi(2)}\right)\left[x_{\pi(3)}, p\left(\bar{y}_{3}\right)\right]
\end{aligned}
$$

for all $\bar{x}_{3}, \bar{y}_{3} \in L^{3}$. We also have (by (10)

$$
\begin{aligned}
& T\left[x_{\pi(2)}, p\left(\bar{y}_{3}\right)\right]=-T\left[p\left(\bar{y}_{3}\right), x_{\pi(2)}\right] \\
= & \sum_{\sigma \in S_{3}}\left[x_{\pi(2)}, y_{\sigma(1)}\right] T\left(y_{\sigma(2)}\right) y_{\sigma(3)}+\sum_{\sigma \in S_{3}} y_{\sigma(1)} T\left[x_{\pi(2)}, y_{\sigma(2)}\right] x_{\sigma(3)} \\
& +\sum_{\sigma \in S_{3}} y_{\sigma(1)} T\left(y_{\sigma(2)}\right)\left[x_{\pi(2)}, y_{\sigma(3)}\right]
\end{aligned}
$$

for all $\bar{y}_{3} \in L^{3}$. Therefore (11) can be written as

$$
\begin{aligned}
& T\left[p\left(\bar{x}_{3}\right), p\left(\bar{y}_{3}\right)\right]=\sum_{\pi \in S_{3}} \sum_{\sigma \in S_{3}}\left[x_{\pi(1)}, y_{\sigma(1)} y_{\sigma(2)} y_{\sigma(3)}\right] T\left(x_{\pi(2)}\right) x_{\pi(3)} \\
& \quad+\sum_{\pi \in S_{3}} \sum_{\sigma \in S_{3}} x_{\pi(1)}\left(\left[x_{\pi(2)}, y_{\sigma(1)}\right] T\left(y_{\sigma(2)}\right) y_{\sigma(3)}+y_{\sigma(1)} T\left[x_{\pi(2)}, y_{\sigma(2)}\right] y_{\sigma(3)}\right. \\
& \left.\quad+y_{\sigma(1)} T\left(y_{\sigma(2)}\right)\left[x_{\pi(2)}, y_{\sigma(3)}\right]\right) x_{\pi(3)} \\
& \quad+\sum_{\pi \in S_{3}} \sum_{\sigma \in S_{3}} x_{\pi(1)} T\left(x_{\pi(2)}\right)\left[x_{\pi(3)}, y_{\sigma(1)} y_{\sigma(2)} y_{\sigma(3)}\right]
\end{aligned}
$$

for all $\bar{x}_{3}, \bar{y}_{3} \in L^{3}$. On the other hand, using

$$
\left[p\left(\bar{x}_{3}\right), p\left(\bar{y}_{3}\right)\right]=-\left[p\left(\bar{y}_{3}\right), p\left(\bar{x}_{3}\right)\right]
$$


we get from the above identity (where for convenience we replace the roles of denotations $\pi$ and $\sigma$ )

$$
\begin{aligned}
& T\left[p\left(\bar{x}_{3}\right), p\left(\bar{y}_{3}\right)\right] \\
= & \sum_{\pi \in S_{3}} \sum_{\sigma \in S_{3}}\left[x_{\pi(1)} x_{\pi(2)} x_{\pi(3)}, y_{\sigma(1)}\right] T\left(y_{\sigma(2)}\right) y_{\sigma(3)} \\
& +\sum_{\pi \in S_{3}} \sum_{\sigma \in S_{3}} y_{\sigma(1)}\left(\left[x_{\pi(1)}, y_{\sigma(2)}\right] T\left(x_{\pi(2)}\right) x_{\pi(3)}+x_{\pi(1)} T\left[x_{\pi(2)}, y_{\sigma(2)}\right] x_{\pi(3)}\right. \\
& \left.+x_{\pi(1)} T\left(x_{\pi(2)}\right)\left[x_{\pi(3)}, y_{\sigma(2)}\right]\right) y_{\sigma(3)} \\
& +\sum_{\pi \in S_{3}} \sum_{\sigma \in S_{3}} y_{\sigma(1)} T\left(y_{\sigma(2)}\right)\left[x_{\pi(1)} x_{\pi(2)} x_{\pi(3)}, y_{\sigma(3)}\right]
\end{aligned}
$$

for all $\bar{x}_{3}, \bar{y}_{3} \in L^{3}$. Comparing so obtained identities we arrive at

$$
\begin{aligned}
0= & \sum_{\pi \in S_{3}} \sum_{\sigma \in S_{3}}\left(\left[x_{\pi(1)}, y_{\sigma(1)} y_{\sigma(2)} y_{\sigma(3)}\right] T\left(x_{\pi(2)}\right)\right. \\
& +x_{\pi(1)}\left(\left[x_{\pi(2)}, y_{\sigma(1)}\right] T\left(y_{\sigma(2)}\right) y_{\sigma(3)}+y_{\sigma(1)} T\left[x_{\pi(2)}, y_{\sigma(2)}\right] y_{\sigma(3)}\right. \\
& \left.+y_{\sigma(1)} T\left(y_{\sigma(2)}\right)\left[x_{\pi(2)}, y_{\sigma(3)}\right]\right)-x_{\pi(1)} T\left(x_{\pi(2)}\right) y_{\sigma(1)} y_{\sigma(2)} y_{\sigma(3)} \\
& \left.+y_{\sigma(1)} T\left(y_{\sigma(2)}\right) y_{\sigma(3)} x_{\pi(1)} x_{\pi(2)}\right) x_{\pi(3)} \\
& +\sum_{\pi \in S_{3}} \sum_{\sigma \in S_{3}}\left(x_{\pi(1)} T\left(x_{\pi(2)}\right) x_{\pi(3)} y_{\sigma(1)} y_{\sigma(2)}\right. \\
& -\left[x_{\pi(1)} x_{\pi(2)} x_{\pi(3)}, y_{\sigma(1)}\right] T\left(y_{\sigma(2)}\right) \\
& -y_{\sigma(1)}\left(\left[x_{\pi(1)}, y_{\sigma(2)}\right] T\left(x_{\pi(2)}\right) x_{\pi(3)}+x_{\pi(1)} T\left[x_{\pi(2)}, y_{\sigma(2)}\right] x_{\pi(3)}\right. \\
& \left.\left.+x_{\pi(1)} T\left(x_{\pi(2)}\right)\left[x_{\pi(3)}, y_{\sigma(2)}\right]\right)-y_{\sigma(1)} T\left(y_{\sigma(2)}\right) x_{\pi(1)} x_{\pi(2)} x_{\pi(3)}\right) y_{\sigma(3)}
\end{aligned}
$$

for all $x_{1}, x_{2}, x_{3}, y_{1}, y_{2}, y_{3} \in L$. Let $s: \mathbb{Z} \rightarrow \mathbb{Z}$ be a mapping defined by $s(i)=$ $i-3$. For each $\sigma \in S_{3}$ the mapping $s^{-1} \sigma s:\{4,5,6\} \rightarrow\{4,5,6\}$ will be denoted by $\bar{\sigma}$. Writing $x_{3+i}$ instead of $y_{i}, i=1,2,3$, in the above identity we can express this relation as

$$
\sum_{i=1}^{6} E_{i}^{i}\left(x_{1}, x_{2}, x_{3}, x_{4}, x_{5}, x_{6}\right) x_{i}=0 .
$$

Since $L$ is 6 -free it follows that $E_{i}=0$ for all $i$. In particular, we have 


$$
\begin{aligned}
0= & E_{6}^{6}\left(x_{1}, x_{2}, x_{3}, x_{4}, x_{5}, x_{6}\right) \\
= & \sum_{\pi \in S_{3}} \sum_{\sigma \in S_{3}}\left(x_{\pi(1)} T\left(x_{\pi(2)}\right) x_{\pi(3)} x_{\bar{\sigma}(4)} x_{\bar{\sigma}(5)}\right. \\
& \quad-\left[x_{\pi(1)} x_{\pi(2)} x_{\pi(3)}, x_{\bar{\sigma}(4)}\right] T\left(x_{\bar{\sigma}(5)}\right)-x_{\bar{\sigma}(4)}\left(\left[x_{\pi(1)}, x_{\bar{\sigma}(5)}\right] T\left(x_{\pi(2)}\right) x_{\pi(3)}\right. \\
& +x_{\pi(1)} T\left[x_{\pi(2)}, x_{\bar{\sigma}(5)]} x_{\pi(3)}+x_{\pi(1)} T\left(x_{\pi(2)}\right)\left[x_{\pi(3)}, x_{\bar{\sigma}(5)}\right]\right) \\
& \left.-x_{\bar{\sigma}(4)} T\left(x_{\bar{\sigma}(5)}\right) x_{\pi(1)} x_{\pi(2)} x_{\pi(3)}\right) .
\end{aligned}
$$

Note that this is also a functional identity which can be rewritten as

$$
\begin{aligned}
0= & \sum_{\pi \in S_{3}} \sum_{\substack{\sigma \in S_{3} \\
\bar{\sigma}(6)=6}} x_{\pi(1)}\left(T\left(x_{\pi(2)}\right) x_{\pi(3)} x_{\bar{\sigma}(4)} x_{\bar{\sigma}(5)}-x_{\pi(2)} x_{\pi(3)} x_{\overline{\sigma(4)}} T\left(x_{\bar{\sigma}(5)}\right)\right) \\
& -\sum_{\pi \in S_{3}} \sum_{\substack{\sigma \in S_{3} \\
\bar{\sigma}(6)=6}} x_{\bar{\sigma}(4)}\left(\left[x_{\pi(1)}, x_{\bar{\sigma}(5)}\right] T\left(x_{\pi(2)}\right) x_{\pi(3)}+x_{\pi(1)} T\left[x_{\pi(2)}, x_{\bar{\sigma}(5)}\right] x_{\pi(3)}\right. \\
& +x_{\pi(1)} T\left(x_{\pi(2)}\right)\left[x_{\pi(3)}, x_{\bar{\sigma}(5)}\right]+x_{\bar{\sigma}(4)} T\left(x_{\bar{\sigma}(5)}\right) x_{\pi(1)} x_{\pi(2)} x_{\pi(3)} \\
& \left.-x_{\pi(1)} x_{\pi(2)} x_{\pi(3)} T\left(x_{\bar{\sigma}(5)}\right)\right)
\end{aligned}
$$

or

$$
\sum_{j=1}^{5} x_{j} F_{j}^{j}\left(x_{1}, x_{2}, x_{3}, x_{4}, x_{5}\right)=0 .
$$

Hence $F_{j}=0$ for all $j$. In particular, we obtain

$$
\begin{aligned}
0 & =F_{1}^{1}\left(x_{1}, x_{2}, x_{3}, x_{4}, x_{5}\right) \\
& =\sum_{\substack{\pi \in S_{3} \\
\pi(1)=1}} \sum_{\substack{\sigma \in S_{3} \\
\sigma(6)=6}}\left(T\left(x_{\pi(2)}\right) x_{\pi(3)} x_{\bar{\sigma}(4)} x_{\bar{\sigma}(5)}-x_{\pi(2)} x_{\pi(3)} x_{\bar{\sigma}(4)} T\left(x_{\bar{\sigma}(5)}\right)\right) .
\end{aligned}
$$

By [3, Corollary 2.7] one gets $T(x)=\lambda x+\mu(x)$ for all $x \in L$, where $\lambda \in C(L)$ and $\mu: L \rightarrow C(L)$. Then substituting this expression to (12) and using [3, Theorem 1.1] it follows that $\mu=0$. Thereby the proof is completed.

We are now in the position to prove Theorem 2.2.

Proof. The complete linearization of (8) gives us (9). Hence, we can apply Theorem 2.3 and conclude that $T$ is indeed a two-sided centralizer unless $R$ is a PI ring (satisfying the standard polynomial identity of degree less than 12). 
Assume now that $R$ is a PI ring. It is well-known that in this case $R$ has a nonzero center (see [13]). Let $c$ be a nonzero central element. Pick any $x \in R$ and set $x_{1}=x_{2}=c x$, and $x_{3}=x$ in (9). Then we arrive at

$$
T\left(6 c^{2} x^{3}\right)=2 c^{2} x T(x) x+4 c x T(c x) x
$$

for all $x \in R$. On the other hand, setting $x_{1}=x_{2}=c$ and $x_{3}=x^{3}$ in (9) we obtain

$$
T\left(6 c^{2} x^{3}\right)=2 c^{2} T\left(x^{3}\right)+2 c T(c) x^{3}+2 c x^{3} T(c)
$$

for all $x \in R$. Comparing so obtained relations we get $2 c\left(2 x T(c x) x-T(c) x^{3}-\right.$ $\left.x^{3} T(c)\right)=0$. Since $R$ is prime it follows that

$$
2 x T(c x) x-T(c) x^{3}-x^{3} T(c)=0
$$

for all $x \in R$. In particular, when $x=c$ we have $2 c^{2} T\left(c^{2}\right)-2 c^{3} T(c)=0$. Therefore

$$
T\left(c^{2}\right)=c T(c) .
$$

The complete linearization of (13) gives us

$$
\begin{aligned}
0= & \sum_{\pi \in S_{3}} 2 x_{\pi(1)} T\left(c x_{\pi(2)}\right) x_{\pi(3)}-\sum_{\pi \in S_{3}} T(c) x_{\pi(1)} x_{\pi(2)} x_{\pi(3)} \\
& -\sum_{\pi \in S_{3}} x_{\pi(1)} x_{\pi(2)} x_{\pi(3)} T(c)
\end{aligned}
$$

for all $x_{1}, x_{2}, x_{3} \in R$. Let $x \in R$. Setting $x_{1}=x$ and $x_{2}=x_{3}=c$ in (15) and using (14) we get

$$
\begin{aligned}
0 & =4 x T\left(c^{2}\right) c+4 c T\left(c^{2}\right) x+4 c T(c x) c-6 T(c) c^{2} x-6 c^{2} x T(c) \\
& =2 c^{2}(-x T(c)-T(c) x+2 T(c x)) .
\end{aligned}
$$

This yields

$$
2 T(c x)=T(c) x+x T(c)
$$

for all $x \in R$. Now setting $x_{1}=x_{2}=x$ and $x_{3}=c$ in (9) we obtain

$$
T\left(6 x^{2} c\right)=2 x T(x) c+2 c T(x) x+2 x T(c) x .
$$

Using (16) we get

$$
3 T(c) x^{2}+3 x^{2} T(c)=2 x T(x) c+2 c T(x) x+2 x T(c) x
$$


for all $x \in R$. A linearization of this relation yields $\left(x_{1}=x, x_{2}=c\right)$

$$
T(c) x+x T(c)=2 c T(x)
$$

for all $x \in R$. By (16) we get

$$
c T(x)=T(c x)
$$

for all $x \in R$. Using (16) and (17) we arrive at

$$
2 T(x y) c=(T(c) x) y+x y T(c)=-x T(c) y+2 T(x) c y+x y T(c)
$$

for all $x, y \in R$. Multiplying (18) on the left by $z \in R$ we get

$$
2 z T(x y) c=-z x T(c) y+2 z T(x) c y+z x y T(c) .
$$

But on the other hand, putting $z x$ instead of $x$ in (18), we obtain

$$
2 T(z x y) c=-z x T(c) y+2 T(z x) c y+z x y T(c) .
$$

Comparing (19) and (20) we get

$$
2 T(z x y) c-2 z T(x y) c=2 T(z x) c y-2 z T(x) c y
$$

for all $x, y, z \in R$. Since $R$ is prime it follows that

$$
T(z x y)=T(z x) y-z T(x) y+z T(x y)
$$

for all $x, y, z \in R$. In particular

$$
T(z c y)=T(z c) y-z T(c) y+z T(c y),
$$

which in turn implies

$$
T(z y) c=T(z) y c-z T(c) y+z T(y) c,
$$

by (17). In this identity write $z x, x \in R$, instead of $z$. Thus we get

$$
T(z x y) c=T(z x) y c-z x T(c) y+z x T(y) c .
$$

Further, using (22) we can write so obtained identity as

$$
T(z x y) c=(T(z) x c-z T(c) x+z T(x) c) y-z x T(c) y+z x T(y) c .
$$

Thus, using (16) and (17), we arrive at

$$
\begin{aligned}
& (T(z x y)-T(z) x y-z T(x) y-z x T(y)) c \\
= & -z(T(c) x+x T(c)) y=-2 z T(x) y c .
\end{aligned}
$$


Since $R$ is prime it follows that

$$
T(z x y)=T(z) x y-z T(x) y+z x T(y)
$$

for all $x, y, z \in R$. Finally, [16, Theorem 2.1] implies the existence of $q \in Q_{s}$ such that

$$
2 T(x)=q x+x q
$$

for all $x \in R$. Consequently, by (8) we get

$$
q x^{3}+x^{3} q=x q x^{2}+x^{2} q x
$$

for all $x \in R$. Thus

$$
\left[[q, x], x^{2}\right]=0 .
$$

A complete linearization of this identity gives us

$$
\sum_{\pi \in S_{3}}\left[\left[q, x_{\pi(1)}\right], x_{\pi(2)} x_{\pi(3)}\right]=0 .
$$

Putting $x_{1}=c$ and $x_{2}=x_{3}=x$ in the above relation it turns out that

$$
0=2\left[[q, c], x^{2}\right]+4[[q, x], x c]=4 c[[q, x], x] .
$$

Thus

$$
[[q, x], x]=0
$$

for all $x \in R$. Using Posner's theorem [12] it follows that $[q, x]=0$ for all $x \in R$. Thus $T$ is a two-sided centralizer. Thereby the proof is completed.

\section{ACKNOWLEDGMENT}

The authors are thankful to Professor Matej Bresar for a useful suggestions concerning the theory of functional identities. The authors would also like to thank the referee for careful reading of the paper and some useful suggestions.

\section{REFERENCES}

1. K. I. Beidar, M. Brešar and M. A. Chebotar, Functional identities revisited: the functional and the strong degree, Comm. Algebra, 30 (2002), 935-969.

2. K. I. Beidar and M. A. Chebotar, On functional identities and d-free subsets of rings I, Comm. Algebra, 28 (2000), 3925-3952.

3. K. I. Beidar and M. A. Chebotar, On functional identities and d-free subsets of rings II, Comm. Algebra, 28 (2000), 3953-3972. 
4. K. I. Beidar and Y. Fong, On additive isomorphisms of prime rings preserving polynomials, J. Algebra, 217 (1999), 650-667.

5. K. I. Beidar, W. S. Martindale III and A. V. Mikhalev, Rings with generalized identities, Marcel Dekker, Inc. New York 1996.

6. K. I. Beidar, A. V. Mikhalev and M. A. Chebotar, Functional identities in rings and their applications, Russian Math. Surveys, 59 (2004), 403-428.

7. M. Brešar, Functional identities: A survey, Contemporary Math., 259 (2000), 93-109.

8. M. Brešar, Jordan derivations on semiprime rings, Proc. Amer. Math. Soc., 104 (1988), 1003-1004.

9. J. Cusak, Jordan derivations on rings, Proc. Amer. Math. Soc., 53 (1975), 321-324.

10. I. N. Herstein, Jordan derivations of prime rings, Proc. Amer. Math. Soc., 8 (1957), 1104-1119.

11. L. Molnár, On centralizers of na $H^{*}$-algebra, Publ. Math. Debrecen, 46 (1995), 89-95.

12. E. C. Posner, Derivations in prime rings, Proc. Amer. Math. Soc., 8 (1957), 10931100 .

13. L. H. Rowen, Some results on the center of a ring with polynomial identity, Bull. Amer. Math. Soc., 79 (1973), 219-223.

14. J. Vukman, An identity related to centralizers in semiprime rings, Math. Univ. Carol., 40 (1999), 447-456.

15. J. Vukman, Centralizers on semiprime rings, Math. Univ. Carol., 42 (2001), 237-245.

16. J. Vukman, I. Kosi-Ulbl and D. Eremita, On certain equations in rings, Bull. Austral. Math. Soc., 71 (2005), 53-60.

17. B. Zalar, On centralizers of semiprime rings, Math. Univ. Carol., 32 (1991), 609-614.

\author{
Joso Vukman \\ Department of Mathematics and Computer Science, \\ PeF, University of Maribor, \\ Koroška cesta 160 , \\ SI-2000 Maribor, \\ Slovenia \\ E-mail: joso.vukman@uni-mb.si \\ Maja Fošner \\ FL, University of Maribor, \\ Mariborska cesta 2, \\ 3000 Celje, \\ Slovenia \\ E-mail:maja.fosner@uni-mb.si
}

